\title{
CONTRIBUCION AL CONOCIMIENTO DE LOS HABITOS ALIMENTICIOS DE LEPIDOCHELYS OLIVACEA Y CHELONIA MYDAS AGASSIZI (REPTILIA, CHELONIIDAE) EN EL PACIFICO MEXICANO
}

\author{
GUSTAVO CASAS-ANDREU Y SAMUEL GOMEZ-AGUTRRE
}

Instituto de Biologia, Universidad Nacional Autónoma de México, México, D.F.

\section{SYNOPSIS}

The results of a study on the feeding habits of L. olivaces and C. mydas agassizi from the Pacific coast, located at $190 \mathrm{~N}$ and $1050 \mathrm{~W}$, are presented. A preference for feeding on crustacea was observed on $\mathrm{L}$. olivacea, proving to be essentially a carnivorous specie. In the case of $\mathrm{C}$. $\mathrm{m}$. agassizi a certain balance between algae and animal feeding was appreciated, beeing the plants in a greater volume and animals more diversified. Related to animal preying C. $\mathrm{m}$. agassizi bucal structure, seems to be adapted toward the retention of animals captured with algae. Apparently there is no competition for food between both species, , given that $\mathrm{L}$. olivacea feeds on organisms from sandy bottoms, while C. m. agassiz feeds on arrecifal or rocky bottoms.

Introducción

En México las tortugas marinas son un recurso natural importante, que en la actualidad está sometido a una intensa explotación, baste citar que la mayor extracción en los últimos afios se obtuvo en 1968 con un total de 13,600 toneladas, habiendo descendido desde entonces, hasta cerca de 2,000 toneladas en 1973 (Márquez, 1976), soportando a esta pesquería principalmente la tortuga golfina ( $L$. olivacea). Los estudios que se han realizado, se han enfocado al conocimiento de la pesquería, hábitos reproductores y migratorios, mientras que los hábitos de alimentación permanecen en gran parte desconocidos. Por lo anterior, nos propusimos realizar una serie de investigaciones de momento en forma preliminar, que nos permitan conocer mejor la alimentación de $L$. olivacea y $C$. m. agassizi, por lo menos de los Estados de Colima y Jalisco en el Pacífico Mexicano.

\section{Antecedentes}

Sobre Lepidochelys olivacea sabemos que es un habitante de aguas marinas protegidas, grandes bahías y lagunas costeras con profundidades relativamente someras (Deraniyagala, 1939, Ernst \& Barbour, 1972 y Márquez et al., 1976). Los últimos autores citados, indican que esta especie se alimenta en fondos marinos de 1 a 50 brazas de profundidad. Deraniyagala (op. cit.), Carr (1952) y Bustard (1972), consideraron esta tortuga como herbívora principalmente. Para Ernst \& Barbour (op. cit.), son tortugas altamente carnívoras, cuya alimentación es a base de peces, cangrejos, caracoles. ostras, holoturias y medusas, ingiriendo ocasionalmente materiales vegetales. Márques et al., (op. cit.), encontrarón en los estómagos de esta tortuga, medusas, 'cangrejos y peces, indicando que en algunos ejemplares se han encontrado exclusivamente langostinos rojos (Pleuroncodes planipes). Zwinenberg (1976), comenta que diversos autores han encontrado en los estómagos de esta espécie, cangrejos, camarones, langostinos, bagres pequefios, caracoles pequeffos y posiblemente medusas.

Por cuanto a Chelonia mydas agassizi se conoce poco de sus hábitos de alimentación, sin embargo, se ha establecido en términos generales, que se alimenta en aguas someras que soportan abundante vegetación (Ernst \& Barbour, 1972), sugeriéndose que estas tortugas no se sumergen más allá de las 12 brazas (Carr, 1967) y en relación con lo anterior Hirth (1971), comenta que las mejores praderas de pastos marinos se encuentran entre esas profundidades. En relación con los hábitos para alimentarse, Smith (1961), propone que en los adultos de esta especie. les papilas coanales se utilizan como filtros de material vegetal. Se ha establecido que en estado adulto la especie presenta un tipo de alimentación preponderantemente herbívora, no siendo contrarias a ingerir animales (Hirth, op. cit.). Previamente sólo se han registrado como materiales ingeridos por esta subespecie en el Pacífico de México, las siguientes algas (Carr, 1961 y Hirth, op. cit.): Gelidium, Sargassum, Rhodymenia, Graci. laria, Griffitsia, Liagora, Ulva, Gratelonpia, Cymodocea y Tha lassia. (?) En C. m. mydas, la subespecie del Atlántico se han registrado moluscos, crustáceos, esponjas, ascidias, briozoarios y equinodermos, además de las algas. Para $C$. $m$. agassizi solo se habían registrado esponjas (Donoso-Barros, 1966).

\section{Materiales y Metodos}

Se colectaron los estómagos de 13 ejemplares de $L$. olivacea que variaron entre 590 y $670 \mathrm{~mm}$ ( 637 promedio), de longitud del carapacho y 9 ejemplares de $C$. $m$. agassizi con tamaños entre
758 y $930 \mathrm{~mm}$ ( 804 promedio), procedentes de capturas comerciales, frente a Playa La Manzanilla $(11 \mathrm{~L}$. olivacea y $7 \mathrm{C} . \mathrm{m}$. agassizi), en mayo de 1974 y frente a Playa de Oro, Golima $(2 \mathrm{~L}$. olivacea y 2 de C. $m$. agassizi), en noyiembro de 1974 (Mapa 1). Se midieron los ejemplares y se res extrajo el estomago para obtener el contenido de los mismos, que se preservó en formol al 5\%. Posteriormente, en el laboratorio, se identificó y cuantifico el material encontrado, ayudado por las obras de Smith, 1944, Dawson, 1952 y 1956 y Abbot \& Hollenberg, 1976, en el reconocimiento de las algas.

\section{Resultados}

Los resultados obtenidos del analísis de los contenidos estomacales de $L$. olivacea se resumen en la Tabla I. De los 13 ejemplares revisados, solo 6 presentaron alimentos en su estómaco. sobresaliendo en la mayoría de éllos, la presencia de crustáceos de diferentes taxa; le sieguen en importancia los restos y conchas de pelecípodos y gasterópodos. En general, los grupos esncontrados forman parte del zoobentos, con excepción de los Scyphozoa (Pelagia sp.) y quetognatos que son componentes del macroplancton (el último grupo representado por Sagitta sp., posiblemente había sido previamente atrapada por la medusa). Otro registro ocasional fué el encuentro de una hoja de mangle (Rhizophora) en un estómago.

A tendiendo a los resultados obtenidos en $C . m$. agassizi, tenemos que de los ejemplares revisados, sólo 7 presentaron contenidos de alimento. Estos resultados se muestran en la Tabla II, que denotan que la mayoría de los estómagos contenían algas, con predominancia de Ulva lactuca y en forma secundaria otras clorofitas y rodofitas. En todos los ejemplares se identificaron animales, habiendo una buena diversidad de crustáceos de tamaños diferentes, abundando los de 2 a $4 \mathrm{~mm}$ de longitud. Otros animales de particular interés fueron los Pyrosoma, encontrados en dos ejemplares de Playa de Oro, que en uno de ellos se encontró como único contenido con un volumen de $200 \mathrm{cc}$. Fué notorio que gran cantidad de conchas de moluscos gasteropodos estaban habitadas por Paguridae. En los contenidos estomacales de ambas especies de tortugas, se reconocieron también nemátodos que no fue posible determinar su procedencia.

\section{Discusion y Conclusiones}

Coincidimos con lo citado por Ernst \& Barbour (1972) y Márquez et al. (1976), por cuanto a que el tipo de alimentación de $L$. olivacea es preponderantemente carnívora y de acuerdo con nuestros resultados, pensamos que existe cierta preferencia por la captura de crustáceos.El tamaño de las presas parece ser importante, ya que encontramos que la mayoría de ellas midieron más de $30 \mathrm{~mm}$ de longitud. El tipo de organismos que contenían los estómagos, con mayor frecuencia, sugiere que esta tortuga tiene hábitos de alimentación con tendencia al bentos de áreas arenosas.

El contenido de los estómagos de C. m. agassizi muestra hábitos de alimentación diversa ya que se observaron tanto vegetales como animales y especialmente éstos úlitimos se encontraron en todos los ejemplares revisados. Se ha señalado a esta especie como preponderantemente herbívora en estado adulto (Carr, 1961 y Hirth, 1971), no obstante, los encuentros de este estudio hacen pensar que el consumo de animales es también importante y dado el tamaño de los encontrados $(10-40 \mathrm{~mm})$, nos sugiere que una de las adaptaciones del aparato bucal (superficie interna de la mandí bula superior acanalada, margen de la mandíbula inferior aserrado 


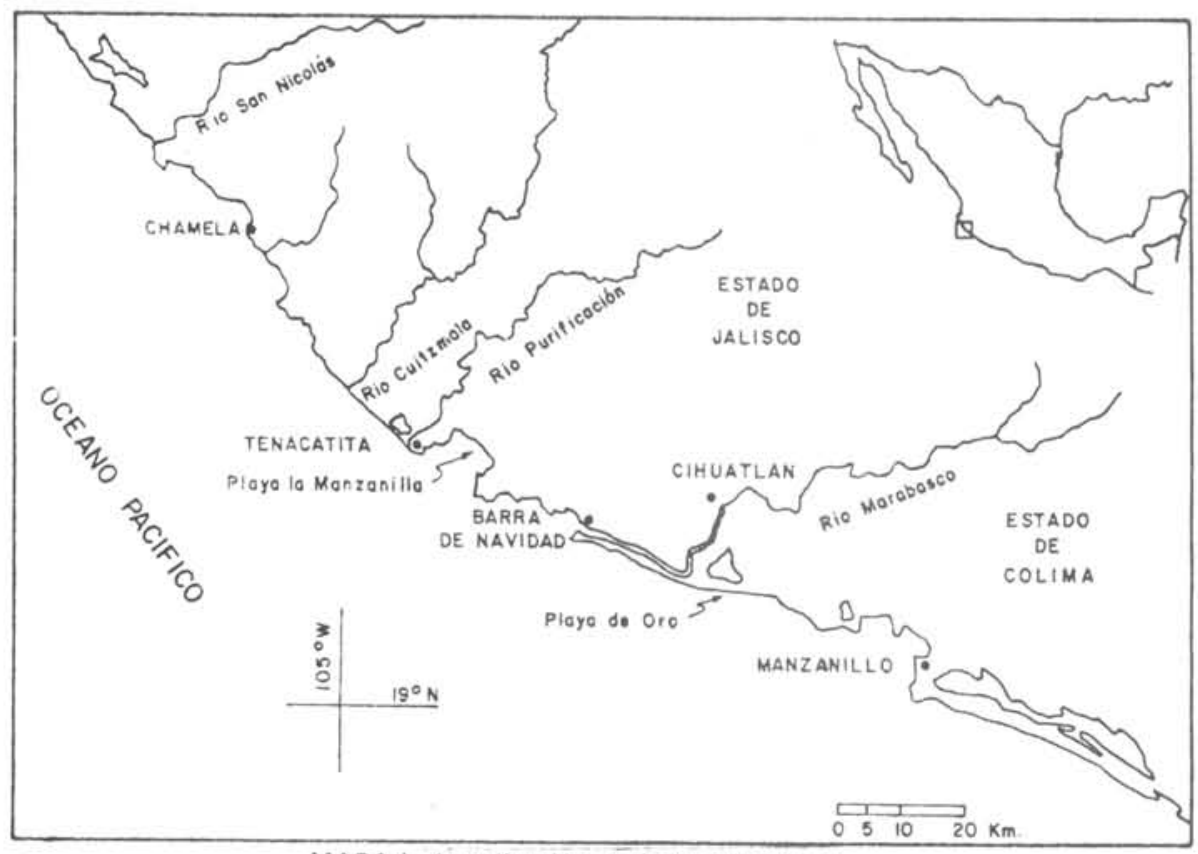

MAPA I. Localización de áreas de captura.

TABLA I - CONTENIDOS ESTOMACALES DE Lepidochelys olivacea CAPTURADAS FRENTE A LAS COSTAS DE COLIMA Y JALISCO, MEXICO

\begin{tabular}{|c|c|c|c|c|c|c|c|c|}
\hline \multirow[b]{2}{*}{$\mathrm{T}$ a $\mathrm{x}$ a } & \multicolumn{6}{|c|}{ Número de ejemplares } & \multirow[b]{2}{*}{ Frecuencia } & \multirow{2}{*}{$\begin{array}{l}\text { Talla } \\
(\mathrm{mm})\end{array}$} \\
\hline & 1 & 2 & 3 & 4 & & 6 & & \\
\hline \multicolumn{9}{|l|}{ Fanerógamas } \\
\hline Rhizophora & & & & & & $\mathrm{x}$ & 1 & 33 \\
\hline Tejidos no identificados & & & & & $\mathrm{x}$ & & 1 & \\
\hline \multicolumn{9}{|l|}{ Celenterados } \\
\hline \multicolumn{9}{|l|}{ Scyphozoa } \\
\hline Pelagia sp juvenil & $\mathrm{x}$ & & & & & & 1 & 30 \\
\hline \multicolumn{9}{|l|}{ Quetognatos } \\
\hline Sagitta sp & $\mathrm{x}$ & & & & & & 1 & 10 \\
\hline \multicolumn{9}{|l|}{ Anélidos } \\
\hline Poliquetos tubícolas & $\mathrm{x}$ & & & & & & 1 & 12 \\
\hline \multicolumn{9}{|l|}{ Moluscos } \\
\hline Pelecypoda & $\mathrm{x}$ & & & & $\mathrm{x}$ & $x$ & 3 & 16 \\
\hline Gasteropoda & $\mathrm{x}$ & & & & & & 1 & 29 \\
\hline \multicolumn{9}{|l|}{ Crustáceos } \\
\hline Isopoda & $\mathrm{x}$ & & & & & & 1 & 6 \\
\hline \multicolumn{9}{|l|}{ Decapoda } \\
\hline \multicolumn{9}{|l|}{ Portunidae } \\
\hline Callinectes sp & & $\mathrm{x}$ & $\mathrm{x}$ & $\mathrm{x}$ & & & 3 & 43 \\
\hline Paguridae & $\mathrm{x}$ & & & & & & 1 & 8 \\
\hline Stomatopoda & & & & & $\mathrm{x}$ & & 1 & 29 \\
\hline Tejidos no identificados & $\mathrm{x}$ & $\mathrm{x}$ & & & $\mathrm{x}$ & $\mathrm{x}$ & 4 & \\
\hline Materiales inorgânicos & & & & & & & & \\
\hline Arenas & & & & & & $\mathrm{x}$ & 1 & \\
\hline Gravillas & & & & & & $x$ & 1 & \\
\hline
\end{tabular}


TABLA II - CONTENIDOS ESTOMACALES DE Chelonia mydas agassizi CAPTURADAS FRENTE A LAS COSTAS DE COLIMA Y JALISCO, MÉXICO

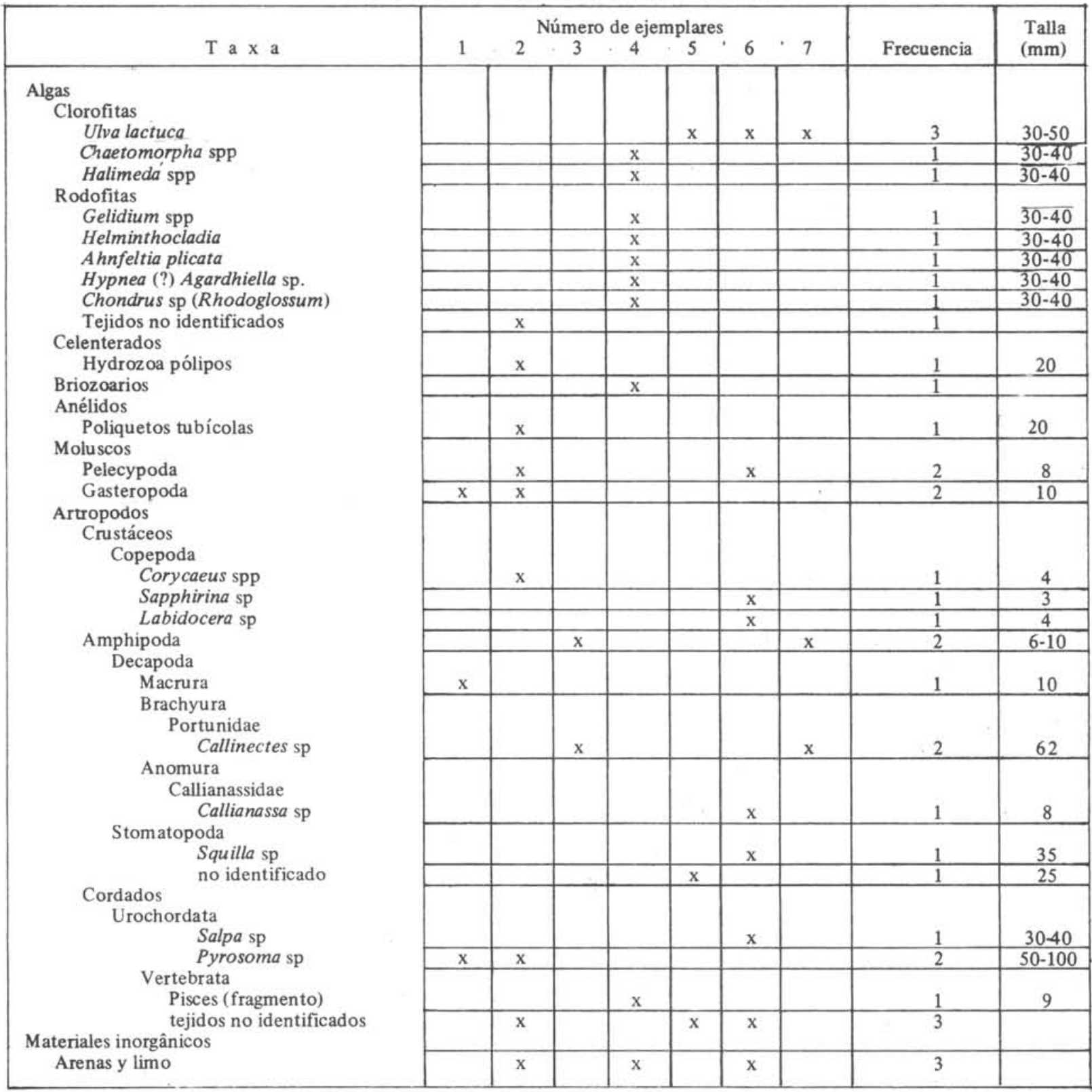

y papilas coanales), de estas tortugas, sería precisamente retener tanto material vegetal como pequeños animales que se encuentran entre éste, por medio de filtración en la boca, función que había sido sugerida por Smith (1961). Se piensa de acuerdo con los resultados, que $C$. $m$. agassizi tiende a alimentarse, predominantemente de bentos de áreas arrecifales o rocosas, sin despreciar el macroplancton (Pelagya sp., Salpa sp. y Pyrosoma sp.) cuando se encuentra disponible.

Al comparar el tipo de alimentos de las dos especies estudiadas, parece observarse que no existe competencia entre ellas por cuanto a sitios y tipo de organismos consumidos, estableciéndose así, alguna otra forma de segregación ecológica.

En el futuro será necesario profundizar más en las observaciones citadas, especialmente áreas de actividad trófica, comportamiento en la selección y captura del alimento en diferentes temporadas del año.

\section{Referencias Bibliográficas}

ABBOTT, 1. A. \& HOLLENBERG, G. J. 1976. Marine algae of California. Stanford, Stanford University Press, 827p., 699 figs.

BUSTARD, R. 1972. Australian sea turtles, their natural history and conservation. London, Collins, 220p.

CARR, A. F. 1952. Handbook of turtles. Ithaca, N. Y., Cornell University Press, 542p.

- ---1961 . Pacific turtle problem. Nat. Hist., 70 (8): 64-71.

- - - - 1967. Caribbean green turtle: imperiled gift of the sea. Natn, geogra. Mag., 131:876-890.
DAWSON, Y. E. 1952. Resumen de las investigaciones recientes sobre algas marinas de la costa pacífica de México, con una sinopsis de la literatura, sinonimia y distribución de las especies descritas. Revta Soc. mex. Hist. nat., 13 (1/4): 97-197.

_- 1956. How to know the seaweeds. Dubuque, lowa, WM C. Brown, 197p., 259 figs.

DERANIYAGALA, P. E. P. 1939. The tetrapod reptiles of Ceylon. Colombo Mus. nat. Hist. Ser., $1: 1-412$.

DONOSO-BARROS, R. 1966. Reptiles de Chile. Santiago, Universidade de Chile, 458p.

ERNST, C. H. \& BARBOUR, R. W. 1972. Turtles of the United States. Kentucky, University Press, 347 p.

HIRTH, H. F. 1971. Synopsis of biological data on the green turtle Chelonia mydas (Linnaeus)' 1958. FAO Fish. Synop. (85): pag. var.

MÅRQUEZ M., R. 1976. Estado actual de la pesquería de tortugas marinas en México, 1974. Serie Infción, Inst. nac. Pesca México, (i46): 1-27.

_._- ; VILLANUEVA O., A. \& REÑAFLORES S., C. 1976. Sinópsis de datos biológicos sobre la tortuga golfina Lepidochelys olivacea (Eschscholtz, 1829) en México. Sinop. Pesca, Inst. nac. Pesca, México, (2): 1-61.

SMITH, G. M. 1944. Marine algae of the Monterrey, California. Stanford. Stanford University Press, 622p. 98 láms.

SMITH, H. M. 1961'. Function of the choanal rakers of the green sea turtle. Herpetologica, 17 (3): 215.

ZWINENBERG. A. J. 1976. The olive ridley, Lepidochelys olivacea (Eschscholtz, 1829): probably the most numerous marine turtle today. Bull. Md her. Soc., 12 (3): 75-95 